\title{
Measurement of Fibrinogen Concentration in the Plasma of Dogs: A Comparison Between Heat Precipitation and Modified Thrombin Clotting Time Method
}

\author{
${ }^{1}$ Labrini V. Athanasiou, ${ }^{1,2}$ Theodoros A. Petanides, \\ ${ }^{1}$ Manolis K. Chatzis and ${ }^{1}$ Manolis N. Saridomichelakis \\ ${ }^{1}$ Department of Medicine, Faculty of Veterinary Medicine, University of Thessaly, Karditsa, Greece \\ ${ }^{2}$ Veterinary Center of Thessaloniki, Thessaloniki, Greece
}

Received 2013-05-26, Revised 2013-06-15; Accepted 2013-07-18

\begin{abstract}
Fibrinogen is a glycoprotein that plays a key role in blood clotting and is also a non-specific indicator of inflammation. The most widely used techniques for measuring plasma fibrinogen concentration include heat precipitation (Millar's technique) and modified thrombin clotting time (Clauss method). Both techniques have been automated in a haematology analyzer (QBC-Vet Autoreader ${ }^{\circledR}$, IDEXX; Millar's technique) and in a newly developed semi-automatic mechanical and optical detection coagulometer (MC1 Plus ${ }^{\circledR}$, DIASys Greiner GmbH; Clauss method). The purpose of this study was to compare the results of the above mentioned techniques for the measurement of fibrinogen concentration in the plasma of dogs. Fibrinogen concentrations were measured with both techniques in the plasma of 85 clinically normal dogs and of 43 dogs with diseases that have been previously associated with dysfibrinogenemia. Passing-Bablock's regression and Bland Altman difference plots were used for the comparison of the results of the two techniques. No correlation was found between the results of the two techniques; thus the two techniques cannot be used interchangeably. Measurement of fibrinogen concentration with the heat precipitation method is more applicable for single sample analysis, while the modified thrombin clotting time method is more practical for analyzing stored frozen samples and thus for research purposes.
\end{abstract}

Keywords: Dog, Plasma, Fibrinogen, Heat Precipitation, Thrombin Clotting Time

\section{INTRODUCTION}

Fibrinogen is an essential component of the haemostatic process, with key roles both in plasmatic clot formation and as a cofactor for the aggregation of platelets (Thrall et al., 2004). Fibrinogen deficiency can occur as an inherited disorder, due to reduced synthesis in liver failure and during consumptive coagulopathies (Mischke et al., 1998), whereas fibrinogen concentrations may be increased in the acute phase of inflammation (Ceron et al., 2005). In dogs, dysfibrinogenemia has been reported during the 5th and 6th week of gestation (Vannucchi et al.,
2002) and in various diseases including leishmaniosis (Petanides et al., 2008), hyperadrenocorticism (Caldin et al., 2009), parvoviral enteritis (Otto et al., 2000), liver cirrhosis (Mischke et al., 1998), chronic renal insufficiency (Mischke, 1997), congestive heart failure (Tarnow et al., 2007), autoimmune hemolytic anemia (Mischke, 1998) and many neoplasms (O'Donnell et al., 1981; Stockhaus et al., 1999).

The most widely used techniques for measuring plasma fibrinogen concentration include heat precipitation and modified thrombin clotting time (Thrall et al., 2004). In the former, fibrinogen Medicine, Faculty of Veterinary Medicine, University of Thessaly, Karditsa, Greece 
concentration is considered equivalent to either the difference in the refractometric total solid concentration before and after heating or to the length of the heat precipitate (Millar's technique) (Millar et al., 1971); Millar's technique has been automated in a haematology analyzer commonly used in veterinary practice (QBCVet Autoreader ${ }^{\circledR}$, IDEXX, Westbrook, Maine, USA). In the latter technique (Clauss method), that has been shown to be rapid, sensitive and precise (Mackie et al., 2002), plasma fibrinogen is quantified by measuring the rate of fibrinogen to fibrin conversion in the presence of thrombin excess (Thrall et al., 2004).

The aim of this study is to compare the results of Millar's technique and Clauss method for measurement of fibrinogen concentration in the plasma of dogs.

\section{MATERIALS AND METHODS}

\subsection{Dogs}

Two groups of dogs were used in the study.

Group A: 85 clinically healthy dogs presented to the Clinic of Medicine, Faculty of Veterinary Medicine, University of Thessaly for routine examination. The majority of these dogs were Mongrels (36.5\%, 31/85), followed by German Shepherds $(31.8 \%, 27 / 85)$, Beagles $(12.9 \%, 11 / 85)$ and the other pure and cross breeds, 56 males and 29 females and their age ranged from 1 to 12 years (median: 4 years).

Group B: comprised of 43 dogs diagnosed with diseases that had been previously associated with dysfibrinogenemia. The majority of these dogs were Greek hounds $(12 / 43 ; 27.9 \%)$, followed by mongrels $(10 / 43 ; 23.3 \%) ; 17(39.5 \%)$ were males and 26 (60.5\%) were non-pregnant females and their age ranged from 1 to 12 years (median: 5.75 years). The final diagnoses of these dogs included leishmaniosis (22 dogs), organspecific bacterial infections such as cystitis, pyometra, discospondylitis and peritonitis (8 dogs), monocytic ehrlichiosis (4 dogs), dirofilariosis due to D. immitis (4 dogs), autoimmune diseases such as pemphigus foliaceous and autoimmune-mediated hemolytic anemia (3 dogs), chronic liver disease (2 dogs), neoplasia (2 dogs) and fever of unknown origin (one dog). The final diagnoses were established based on history, physical examination, laboratory examinations and imaging studies that were considered appropriate based on the attending clinician's discretion; more than one diagnosis was established for 3 dogs.

\subsection{Sampling}

Blood samples were collected by jugular venipuncture for preventative (group A) or diagnostic (group B) examinations. After owner's informed consent, a part of the blood was transferred into one tube containing $3.2 \%$ sodium citrate and another containing potassium EDTA. Samples were visually inspected and they were rejected if clots were apparent. Tubes containing sodium citrate were centrifuged for $10 \mathrm{~min}$ at $3000 \mathrm{~g}$ and plasma was separated into two aliquots.

\subsection{Stability Testing}

For logistic reasons, plasma fibrinogen concentrations had to be measured with the Clauss method more than two hours after measurement with the Millar's technique. Therefore, a preliminary stability study was performed (Jensen and Kjelgaard-Hansen, 2006). Sample stability in terms of fibrinogen concentration measured with the Clauss method was examined after freezing at $-20^{\circ} \mathrm{C}$ eight aliquots from two pools of fresh citrated plasma. One pool consisted of plasma from ten dogs with a mean fibrinogen concentration about $200 \mathrm{mg} / \mathrm{dL}$ and the other one from ten dogs with an at least double mean plasma fibrinogen concentration. Stability was assessed by measuring plasma fibrinogen concentration immediately after sample pooling and $24 \mathrm{~h}, 48 \mathrm{~h}, 72 \mathrm{~h}, 7$ days, 14 days, 21 days and 28 days after freezing.

\subsection{Sample Analysis}

Plasma fibrinogen concentration was measured with the Millar's technique using the QBC-Vet Autoreader ${ }^{\circledR}$, according to the manufacturer's instructions. Briefly, a QBC-tube was filled with EDTA-anticoagulated blood, centrifuged for $5 \mathrm{~min}$ at the IDEXX VetCentrifuge ${ }^{\circledR}$, heated to $56^{\circ} \mathrm{C}$ in the instrument's heating box, centrifuged for $5 \mathrm{~min}$ and placed into the automatic analyzer.

Plasma fibrinogen concentration was measured with the Clauss method using the MC1 Plus ${ }^{\circledR}$ (DiaSys Greiner GmbH, Flacht, Germany) semiautomatic coagulometer, according to the manufacturer's instructions. Citrated plasma diluted 1:10 in imidazole buffer solution was left to clot in the presence of excess thrombin. Under such conditions, the fibrinogen concentration is inversely proportional to the clotting time. A calibration curve was prepared from a fibrinogen reference standard, provided by the manufacturer, plotted on log-log paper and was used to determine the fibrinogen concentration in the test sample. 


\subsection{Statistical Analysis}

To assess the influence of the time of storage at$20^{\circ} \mathrm{C}$ on the concentration of fibrinogen in canine citrated plasma measured with the Claus method, results obtained at different time points were compared with the one way analysis of variance for repeated measurements, followed by Bonferroni's correction, with the value at time 0 used a reference. The statistical software package SPSS ${ }^{\circ}$ v.20 for Windows was used for data processing. Plasma fibrinogen concentrations that were measured with the two techniques were compared using PassingBablok's regression and Bland-Altman difference plots, two methods where linear regression assumptions are not expected to be met (Bland and Altman, 1986; Jensen and Kjelgaard-Hansen, 2006). Comparisons were performed using the software MedCalc v. 12.3.0.0, (MedCalc Software, Ostend, Belgium).

\section{RESULTS}

Time of storage did not significantly affect fibrinogen concentration ( $\mathrm{p} \geq 0.89$ for all comparisons) and fibrinogen concentration in citrated plasma that was stored at $-20^{\circ} \mathrm{C}$ was found to be stable for 28 days.

No correlation was found between the results of the two techniques. The scatterplot comparing the results obtained by the two techniques in 85 group a and the 43 group B dogs is shown on Fig. 1. The $95 \%$ Confidence Interval (CI) of the intercept $\mathrm{A}$ and the $95 \%$ CI of the slope B were-856.12 to-179.5 and 2.44 to 5.52 respectively. Similarly, visual inspection of the Bland-Altman difference plot (Fig. 2) shows a random variation of the differences in fibrinogen concentrations measured with the two techniques.

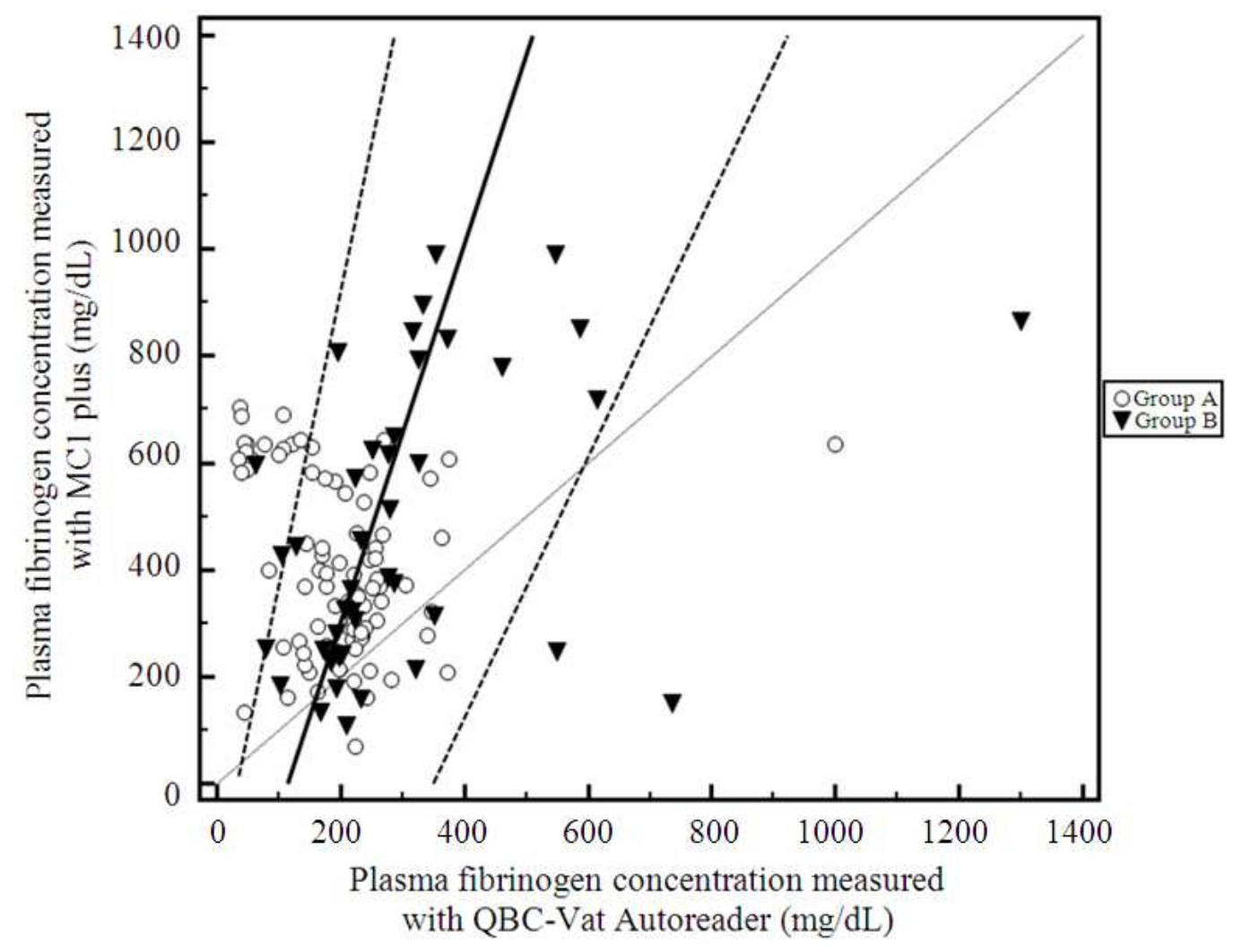

Fig. 1. Passing-Bablock scatterplot comparing fibrinogen concentrations measured with QBC-Vet Autoreader ${ }^{\circledR}$ and $\mathrm{MC} 1$

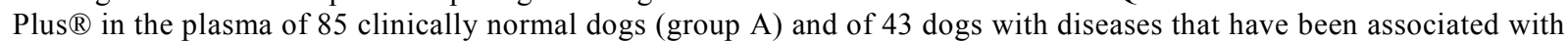
dysfibrinogenemia (group B). The thin solid line is the line of identity, the thick solid line is the line of regression and the dashed lines represent the $95 \%$ confidence interval for the regression line. The equation of the regression was fibrinogen concentration measured with $\mathrm{MC1}$ Plus ${ }^{\circledR}=-409.595+3.554 *$ fibrinogen concentration measured with QBC-Vet Autoreader ${ }^{\circledR}$ 


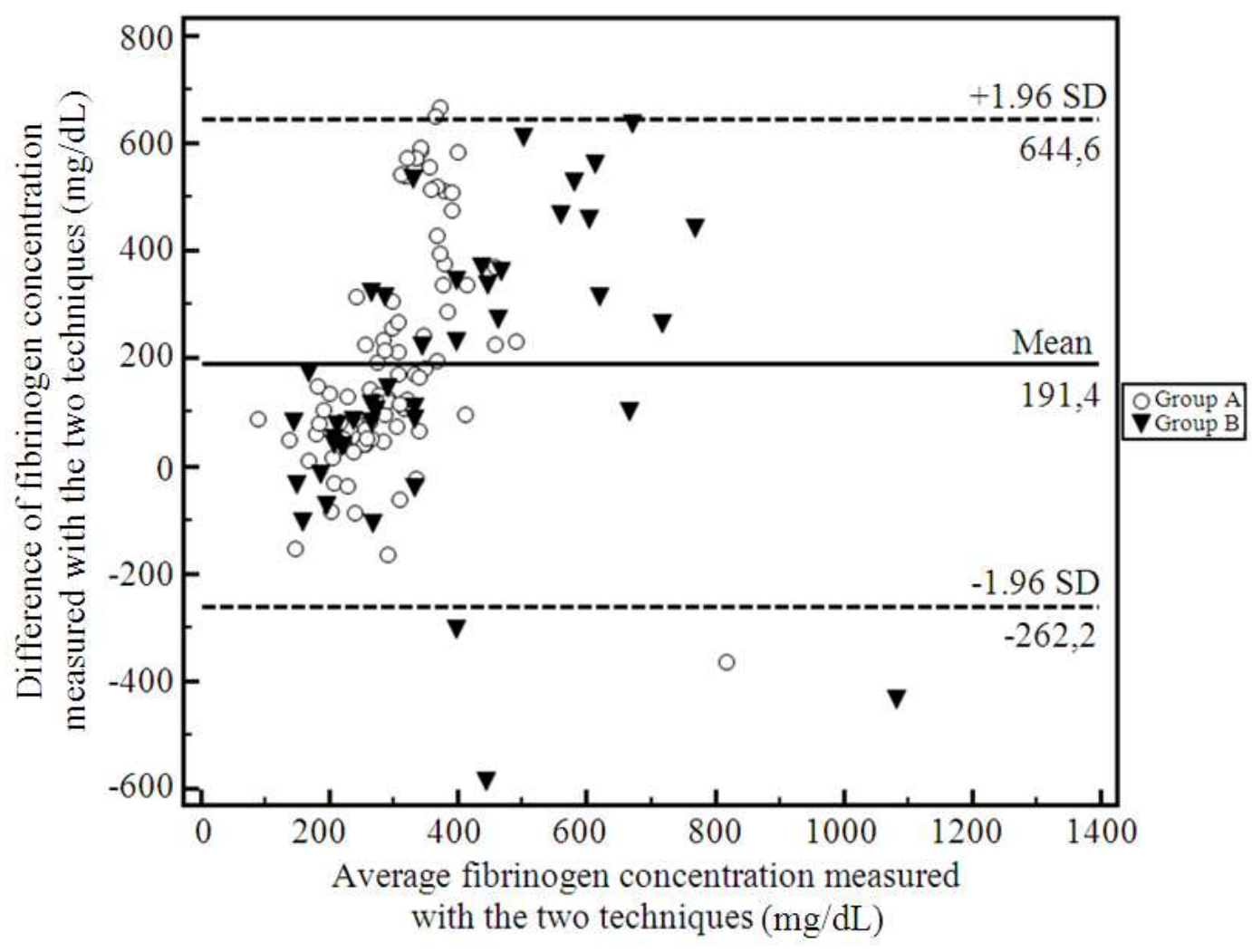

Fig. 2. Bland-Altman plot showing the differences of the fibrinogenn concentrations measured with QBC-Vet Autoreader ${ }^{\circledR}$ and MC1 Plus ${ }^{\circledR}$ in the plasma in the plasma of 85 clinically normal dogs (group A) and of 43 dogs with diseases that have been associated with dysfibrinogenemia (group B) plotted against the average of the two techniques. The thick solid line represents the mean difference of the two techniques and the dashed lines represent the limits of agreement which are defined as the mean difference $\pm 1.96 *$ Standard Deviation (SD) of the difference

Table 1. Frequency of decreased and of increased plasma fibrinogen concentrations measured with QBC-Vet Autoreader ${ }^{\circledR}$ in clinically normal dogs (group A) and in dogs with diseases that have been associated with dysfibrinogenemia (group B)

\begin{tabular}{lll} 
& QBC-Vet Autoreader $\mathbb{R}\left(\right.$ reference interval: $\left.100-250 \mathrm{mg} \mathrm{dL}^{-1}\right)$ \\
Dogs & Increased fibrinogen concentration & Decreased fibrinogen concentration \\
\hline Group A & $18 / 85(21.2 \%)$ & $12 / 85(14.1 \%)$ \\
Group B & $20 / 43(46.5 \%)$ & $3 / 43(6.98 \%)$ \\
\hline
\end{tabular}

Using the manufacturer's reference interval of 100-250 $\mathrm{mg} \mathrm{dL}^{-1}$ (IDEXX, 2006) for canine plasma fibrinogen concentration measured with the Millar's technique using the QBC-Vet Autoreader ${ }^{\circledR} 30 / 85$ (35.3\%) group A and $23 / 43(53.5 \%)$ group B dogs had abnormal plasma fibrinogen concentrations (Table 1). In particular, the concentration of fibrinogen was above the upper reference limit in $11(50 \%)$ of the 22 dogs with leishmaniosis, respectively and in $4(50 \%)$ of the 8 dogs with organ-specific bacterial infections, respectively.

\section{DISCUSSION}

Measurement of plasma fibrinogen concentration is useful in the evaluation of coagulopathies and inflammatory disorders. Different techniques and analyzers have been used for measurement of plasma fibrinogen concentration in various animal species, but none of them can be considered as gold standard (Mischke et al., 2000).

In the present study we compared two techniques in order to determine whether they can be used 
interchangeably for measurement of fibrinogen concentration in canine plasma (Bland and Altman, 1986). The disagreement between the results of the two techniques could be attributed to the different principles used to estimate fibrinogen concentration. Heat precipitation measures the total amount of plasma proteins that precipitate at $56^{\circ} \mathrm{C}$ and these proteins include mainly, but not exclusively, the fibrinogen. On the contrary, Clauss method measures the "activity" of the clotting cascade in term of fibrin production in excess of thrombin, which is subjected to interference by other substances, besides fibrinogen, that are involved in the process of coagulation (Mischke et al., 2000).

A stability study was performed, according to the recommendations of the American Society of Veterinary Clinical Pathology (Jensen and Kjelgaard-Hansen, 2006) and showed that citrated canine plasma can be stored for at least 28 days at-200C prior to measurement of fibrinogen concentration with the Clauss method using the MC1 Plus ${ }^{\circledR}$ analyzer. This may be an advantage of this method compared to Millar's technique using the QBC-Vet Autoreader ${ }^{\circledR}$. No stability data for the latter are available from the manufacturer since measurement of fibrinogen usually follows Complete Blood Count (CBC) and is done soon after blood sampling. Therefore, measurement of fibrinogen concentration with the QBCVet Autoreader ${ }^{\circledR}$ is more applicable for single sample analysis, while the $\mathrm{MC1}$ Plus ${ }^{\circledR}$ analyzer is more practical for analyzing stored frozen samples and thus for research purposes. The simultaneous $\mathrm{CBC}$ and measurement of fibrinogen concentration reduces the cost of the analysis while the comparatively less time to get the results is another advantage of QBC-Vet Autoreader ${ }^{\circledR}$. The assessment of the applicability of the two methods, including the real cost per analysis, the cost of analyzer and the operator training required, seems to be rather subjective and quite variable in different countries (Jensen and Kjelgaard-Hansen, 2006).

Fibrinogen concentrations in the plasma of clinically normal dogs differed widely and $37.6 \%$ of them felt outside the reference interval suggested by the manufacturer of QBC-Vet Autoreader $\AA$, raising doubts on its validity. This finding may be attributed to the methodology that has been used for the establishment of the reference interval and/or to the criteria for the definition of the "healthy" canine population.

The frequency of hyperfibrinogenemia was 25 and $50 \%$ in dogs with organ-specific bacterial infections and plasma fibrinogen concentrations were within reference intervals in many other dogs with various inflammatory conditions. Although fibrinogen is an acute phase protein it is not as sensitive as other acute phase reactants which show a higher and more rapid response to inflammatory stimuli; therefore, it is not surprising that dysfibrinogenemia due to coagulopathies rather than hyperfibrinogenemia due to inflammation is more frequently reported in veterinary medicine (Ceron et al., 2005).

\section{CONCLUSION}

As methods for the measurement of fibrinogen concentration in the plasma are becoming readily available their use in clinical diagnosis is likely to expand, the results of the present study show that the interpretation of results should be done with respect to the technique that has been used and its specific reference interval.

\section{ACKNOWLEGMENTS}

This project was partially funded by the Research Committee of the University of Thessaly and partially self-funded.

\section{REFERENCES}

Bland, J.M. and D.G. Altman, 1986. Statistical methods for assessing agreement between two methods of clinical measurement. Lancet, 327: 307-310. DOI: 10.1016/S0140-6736(86)90837-8

Caldin, M., S. Tasca, E. Carli, S. Bianchini and T. Furlanello et al., 2009. Serum acute phase protein concentrations in dogs with hyperadrenocorticism with and without concurrent inflammatory conditions. Vet. Clin. Pathol., 38: 63-68. DOI: 10.1111/j.1939-165X.2008.00087.x

Ceron, J.J., P.D. Eckersall and S. Martynez-Subiela, 2005. Acute phase proteins in dogs and cats: Current knowledge and future perspectives. Vet. Clin. Pathol., 34: $\quad 85-99 . \quad$ DOI: $10.1111 /$ j.1939165X.2005.tb00019.x

IDEXX, 2006. IDEXX Vet Autoread Hematology Analyzer. IDEXX Laboratories, USA.

Jensen, A.L. and M. Kjelgaard-Hansen, 2006. Method comparison in the clinical laboratory. Vet. Clin. Pathol., 35: 276-286. DOI: 10.1111/j.1939165X.2006.tb00131.x

Mackie, I.J., A.S. Lawrie, S. Kitchen, P.J. Gaffney and D. Howarth et al., 2002. A performance evaluation of commercial fibrinogen reference preparations and assays for Clauss and PT-derived fibrinogen. Thrombosis Haemostasis, 87: 997-1005. 
Millar, H.R., J.G. Simpson and A.L. Stalker, 1971. An evaluation of the heat precipitation method for plasma fibrinogen estimation. J. Clin. Pathol., 24: 827-830. PMID: 5003786

Mischke, R., 1997. Changes in hemostasis in dogs with chronic renal insufficiency. Berl. Munch Tierarztl. Wochenschr, 110: 445-450. PMID: 9451845

Mischke, R., 1998. Hemostatic disorders as a complication of autoimmune hemolytic anemia in dogs. Dtsch Tierarztl. Wochenschr, 105: 13-16. PMID: 9499627

Mischke, R., D. Menzel and H. Wolling, 2000. Comparison of different methods to measure fibrinogen concentration in canine plasma with respect to their sensitivity towards the fibrinogen degradation products $\mathrm{X}, \mathrm{Y}$ and D. Haemostasis, 30: 131-138. DOI: 10.1159/000022535

Mischke, R., D. Pohle, H.A. Schoon, M. Fehr and I. Nolte, 1998. Alterations of hemostasis in liver cirrhosis of the dog. Dtsch. Tierarztl. Wochenschr, 105: 43-47. PMID: 9528205

O’Donnell, M.R., S.J. Slichter, P.L. Weiden and R. Storb, 1981. Platelet and fibrinogen kinetics in canine tumors. Cancer Res., 41: 1379-1383.

Otto, C.M., T.M. Rieser, M.B. Brooks and M.W. Russell, 2000. Evidence of hypercoagulability in dogs with parvoviral enteritis. J. Am. Vet. Med. Assoc., 217: 1500-1504. DOI: 10.2460/javma.2000.217.1500
Petanides, T.A., A.F. Koutinas, M.E. Mylonakis, M.J. Day and M.N. Saridomichelakis et al., 2008. Factors associated with the occurrence of epistaxis in natural canine leishmaniasis (Leishmania infantum). J. Vet. Internal Med., 22: 866-872. DOI: 10.1111/j.1939-1676.2008.0129.x

Stockhaus, C., B. Kohn, R. Rudolph, L. Brunnberg and U. Giger, 1999. Correlation of haemostatic abnormalities with tumour stage and characteristics in dogs with mammary carcinoma. J. Small Anim. Pract., 40: 326-331. DOI: 10.1111/j.1748-5827.1999.tb03090.x

Tarnow, I., T. Falk, A. Tidholm, T. Martinussen and A.L. Jensen et al., 2007. Hemostatic biomarkers in dogs with chronic congestive heart failure. J. Vet. Intern Med., 21: 451-457. DOI: 10.1111/j.1939-1676.2007.tb02989.x

Thrall, M.A., D.C. Baker and E.D. Lassen, 2004. Veterinary Hematology and Clinical Chemistry. 1st Edn., Wiley, Oxford, ISBN-10: 0781768500, pp: 618 .

Vannucchi, C.I., R.M. Mirandola and C.M. Oliveira, 2002. Acute-phase protein profile during gestation and diestrous: Proposal for an early pregnancy test in bitches. Anim. Reprod. Sci., 74: 87-99. DOI: 10.1016/S0378-4320(02)00160-4 